\title{
Volunteers in end-of-life care for non-oncological Patients - Are there Distinctions?
}

\section{Lena Deiseroth ${ }^{1}$, Gabriella Marx ${ }^{1,2}$, Maximiliane Jansky ${ }^{1}$, Christian Banse ${ }^{1}$,}

Kathrin Heiß ${ }^{1}$, Friedemann Nauck ${ }^{1}$

${ }^{1}$ Clinic for Palliative Medicine, University Medical Center Goettingen, Goettingen, Germany

${ }^{2}$ Department of General Practice / Primary Care, University Medical Center Hamburg-Eppendorf, Hamburg, Germany

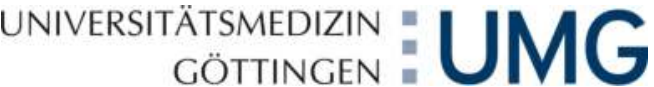

\section{Background and Aim}

- Volunteers have a fundamental role in end-of-life care and are an essential part of multi-professional palliative care today.

- Non-oncological diseases are characterised as ,long term limitations with intermittent serious episodes' ${ }^{\prime 1}$. Therefore the continuous integration of non-oncological patients (NOPs) into palliative care may create distinct challenges and problems for volunteers, when supporting these patients and their relatives compared to oncological patients (OPs).

- Aim: To assess volunteer support for NOPs and potential distinctions to OPs in Germany.

\section{Design and Methods}

- Quantitative study design with qualitative elements

- Online survey to coordinators of volunteer services throughout Germany (212 participants)

- Themes

- NOPs-support in the past compared to OPs-support

- Importance of factors such as age, disease and family status in establishing volunteer care

- Analysis using descriptive statistics and qualitative content analysis

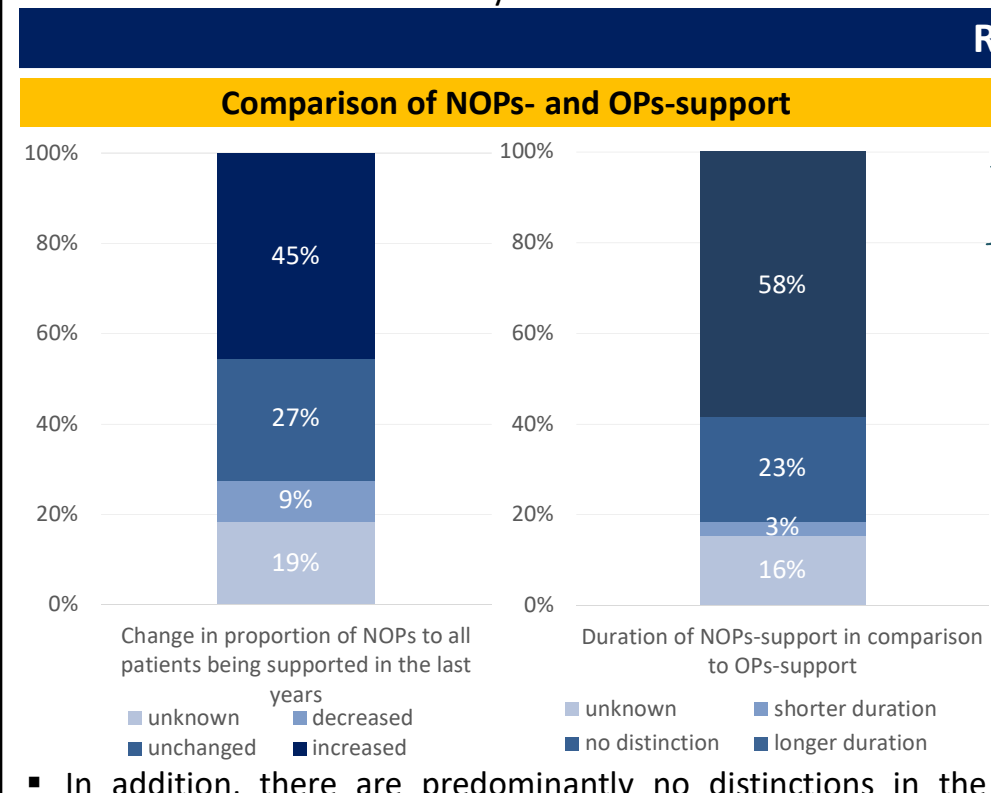

- In addition, there are predominantly no distinctions in the volunteer readiness $(75 \%)$ and number of inquiries $(73 \%)$ in NOPs-support.

\section{Training and Information for volunteers}

- In almost all cases (96\%) the volunteers get informed about the patient's disease. (However, some do not want to know the patient's diagnosis e.g. to contact the patient unprejudiced.)

- Specific training of volunteers for NOPs-support (e.g. about dementia) is important to $47 \%$ of the coordinators whereas $42 \%$ do not consider it important.

,The volunteer training includes various, also nononcological disease patterns like ALS [amyotrophic lateral sclerosis] and dementia.'

\section{Results}

,Especially in chronical courses of disease [it is] a difficult
question when to start patient support by volunteers.'
Study participant 51
'In most cases the moment of inquiry or the progress
of disease determines the duration of the support.'

Impact of patient characteristics in arranging voluntary support $100 \%$

$80 \%$

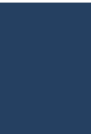

$50 \%$

$60 \%$

$40 \%$

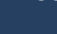

\section{$20 \%$}

$0 \%$
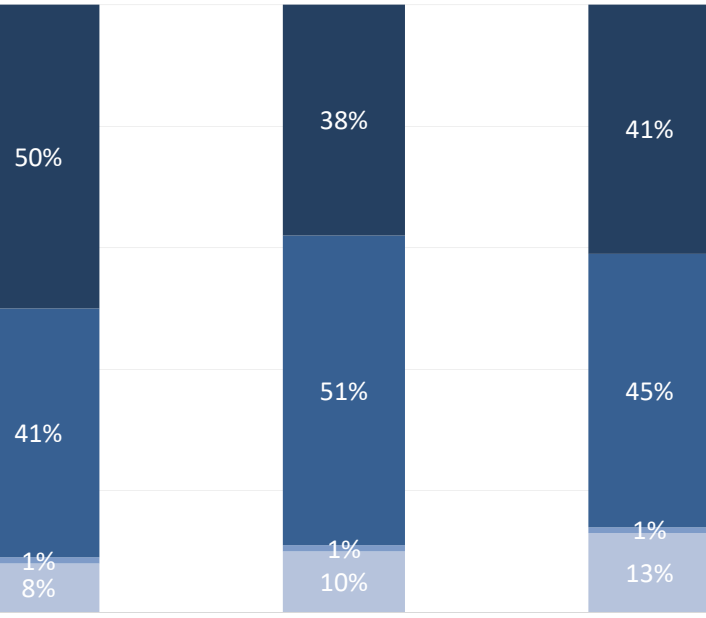

Intensity of symptoms Possibility for communication Expected duration of support no specification no influence $\mathbf{a}$ low/rather low influence $\mathbf{m i g h} /$ rather high influence

,The intensity of symptoms is an important factor. Practical experience shows that the volunteers do not shy away from complex symptoms and feel prepared because of good communication between coordinators and volunteers.'

\section{Conclusions and Take home message}

- Close contact between coordinators and volunteers supporting NOPs is needed to give the volunteers a feeling of safety when dealing with prolonged support.

- The increase of NOPs with prolonged supports compared to OPs might lead to a lack of volunteers for urgent requirements (especially for smaller volunteer services with little resources).

- In Germany volunteers get further education for supporting patients. Their main role is ,being-there': Volunteers support patients to cut their own path and find themselves in their last phase of life ${ }^{2}$.

- Specific training for volunteers focusing on longer support, unique requirements and disease specific factors of NOPs might benefit the satisfaction for both, patients and volunteers.

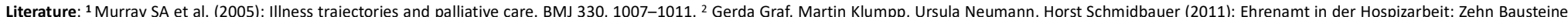
zur Erarbeitung eines Leitbildes 